\title{
Parietal Foramen as A Landmark to Locate Intracranial Vascular and Parenchymal Structures
}

Pedro Brainer-Lima ( $\sim$ pedro.brainerlima@ufpe.br)

Federal University of Pernambuco

Alessandra Brainer-Lima

Departament of Radiology, PROCAPE

Maria Rosana Ferreira

Federal University of Pernambuco

Paulo Brainer-Lima

Hospital da Restauração

Marcelo Valença

Federal University of Pernambuco

\section{Research Article}

Keywords: parietal foramina, landmarks, vascular

Posted Date: December 7th, 2020

DOI: https://doi.org/10.21203/rs.3.rs-114662/v1

License: (c) (i) This work is licensed under a Creative Commons Attribution 4.0 International License.

Read Full License 


\section{Abstract}

The aim of this study was to define the location of the parietal foramina (PF) with reference to skull landmarks and correlate the PF with cerebral and vascular structures to optimize neurosurgical procedures in the intracranial compartment. Two hundred and thirty-eight parietal bones studied by magnetic resonance imaging (MRI) of 119 patients were reviewed. The cephalometric points, inion, bregma, sagittal suture and lambda were used as anatomical references to locate the PF and define its anatomical relationships to parenchymal cerebral structures, especially some eloquent areas. The PF was identified in the MRI in 83 of the 119 individuals (69.7\%) and was located at an average distance of $9.5 \pm$ $0.8 \mathrm{~cm}$ (mean \pm SD) posteriorly and $0.9 \pm 0.3 \mathrm{~cm}$ laterally to the Bregma. In over $90 \%$ of cases, the PF was located within a $2 \mathrm{~cm}$ radius of the bregma-PF distance's mean value. Surgeons operating in the parietal region should be aware of the frequency of PF (69.7\%), its location (superolateral to lambda) and its stable relationship with underlying anatomical structures. $88 \%$ of the 62 left PF's were situated within $1 \mathrm{~cm}$, laterally to the left margin of the superior sagittal sinus (SSS). $60 \%$ of the right PF were situated within $1.3 \mathrm{~cm}$ laterally from the right margin of the SSS, while $40 \%$ were directly above the SSS. We propose that the PF should be used as the reference for the superior sagittal sinus during its course through the parietal lobe, as its constancy overtakes other commonly used landmarks (sagittal suture and midline). In conclusion, clinicians should be aware of the PF to both avoid iatrogenic injury to an emissary vein that courses through it that can lead to air embolism and as a guide to maneuvering through the parietal region.

\section{Introduction}

The parietal foramen (PF) is a naturally occurring fine canal that perforates the posterior region of the parietal bone, positioned 2-5 millimeters laterally to the sagittal suture ${ }^{1-7}$. The PF is site for both an arterial anastomosis between the middle meningeal and scalp arteries and an emissary vein that bridges intracranial venous vessels (i.e. superior sagittal sinus and diploic venous structures) to the scalp venous system $^{3,8}$. This vascular relation is determinant for PF's anatomy and localization. The PF may be absent, occur unilaterally or more commonly, bilaterally $(>50 \%)$. Prior literature has shown the PF is present in $60-$ $80 \%$ of dry skulls examinations $2,5,9,10$.

It is believed that the valveless emissary vein that courses through the PF plays both a vascular and a physiological function in controlling intracranial temperature and pressure, given its ability to instill a bidirectional blood flow from the intracranial space to the extracranial one or vice versa ${ }^{11-13}$. A study has shown that during hyperthermia, scalp to brain blood flow is increased, while during hypothermia, either the flow stops or an opposite one is established ${ }^{12}$. It appears that only the extant humans present relatively high frequencies of the parietal foramen which in conjunction to skull thinning, was probably motivated by evolutionary epigenetic components ${ }^{13}$. This means that the increase in parietal and other foramina were motivated by practical factors, such as hominid bipedality, brain enlargement, 
orthognathy ${ }^{14}$ and a need for brain cooling as other structures (i.e. Occipital/Marginal Sinuses) decreased in the frequency ${ }^{13}$.

PFs are distinguishable both in radiology studies and in vivo ${ }^{3}$. Surgeons could use PF's location to estimate anatomical relationships to parenchymal structures, especially eloquent areas (e.g. angular gyrus) as to place the craniotomy window accordingly or use PF's position to coordinate maneuvers around the superior sagittal sinus (SSS), while avoiding injury, which albeit infrequent can be fatal ${ }^{6}$. Improper craniotomies in the parietal region lacking awareness of the PF, can affect the emissary vein and be a source of air embolism ${ }^{4}$. Thus, clinicians should consider the presence of the PF and its anastomosis to navigate the parietal region effectively and avoid iatrogenic injury to vascular structures.

While the PF has been investigated in prior literature, there are scarce reports on PF's anatomical correlations, resulting in subpar information on this foramen. Previous studies diverged in landmarks used to locate the PF, applying either inion ${ }^{6}$, bregma ${ }^{7}$ or lambda $^{8}$. The lack of a standardized localization method difficulties both accurate clinical use and future research of the PF. Additionally, as prior research were mostly restricted to the use of cadavers, establishing correlations between PF and parenchymal structures was not achieved.

Inion, bregma and lambda are anatomical landmarks that can be used for surgical planning. These landmarks are accessible, static, and thus a comparison between those three landmarks is necessary for pinpointing which is the most suitable point of reference for identifying the PF, which in turn can help accurately identify deep nearby eloquent structures (e.g. angular gyrus, central sulcus and SSS). This study focusses on using MRI to determine PF's reliability as a landmark for identifying the underlying parenchymal and venous structures, helping clinicians safely and accurately maneuver through the parietal lobe and SSS.

The presented data are mean \pm SD.

\section{Results}

Most measurements conformed to a normal distribution, except those related to distances from SSS margin and left or right PF (parameter \#13). Laterality difference between the left and right sides of the hemiface was found in parameter \#13 and specified parameters in the Table 1, alongside their respective values and displayed in Fig. 2. All the identified PFs perforated the entire cranium as single channel, each with a single emissary vein, delineating an intensely enhanced straight or curvilinear line passing through the foramen (Fig.1A, C).

The PF was present in 83 of 119 studied patients (69.7\%). Both parietal bones of each patient were reviewed individually, totalizing 166 parietal bones, of which $122(73.4 \%)$ had PFs. It was observed that $23 \mathrm{PFs}$ were on the left hemicranium, 21 on the right and 39 were bilateral cases. Figure 4 displays the 122 foramens distribution. All PFs were located laterally to the sagittal suture and anteriorly to lambda. 
There was not a significant difference in PF's relation to landmarks between male and females $X^{2}(1$, $\mathrm{N}=83)=4.44, \mathrm{p}=0.108$.

The average horizontal distance from the obelion to the PF (\#2) had a value of $8.86 \pm 3.07 \mathrm{~mm}$. The vertical distance from obelion to lambda (\#1) had a value of $35.28 \pm 6.9 \mathrm{~mm}$, to bregma (\#4) $94.91 \pm$ $8.32 \mathrm{~mm}$, and to inion (\#5) $102.8 \pm 13,93 \mathrm{~mm}$. The average horizontal component (\#11) of the distance from PF to the angular gyrus was $75.11 \pm 12.1 \mathrm{~mm}$, while the vertical component (\#3) was split into left and right, given laterality significance $(p<0.010)$ was split into left and right, $25.62 \pm 10.57 \mathrm{~mm}$ and $34.93 \pm 10.05 \mathrm{~mm}$, respectively. Pearson's correlation tests between the distances (\#1 and \#6, and \#5, and $\# 4)$ were analyzed. Parameter \#1 was negatively correlated to \#6 $(p<0.05)$, and to \#4 $(p<0.001)$ and positively correlated to \#5 ( $\mathrm{p}<0.05)$.

The relationship between the PF and SSS (parameter \#13) was delineated in MRI axial slices, at the height of the PF, on the posterior third of the sagittal suture (Fig. 3). The distance from the left PF to the left margin of the SSS averaged $3.73 \pm 3.61 \mathrm{~mm}$, while the distance from the right PF to the right margin of the SSS had a value of $3.57 \pm 3.14 \mathrm{~mm}$. The mean width of the SSS was $12.06 \pm 3.47 \mathrm{~mm}$.

\section{Discussion}

$\mathrm{PF}$ is a recognizable anatomical landmark that can help pinpoint nearby eloquent structures, both in vivo and in MRI studies ${ }^{15-17}$. This can cut back surgery time as it increases accuracy, especially during emergency surgeries and in hospitals that lack access to a reliable neuronavigation equipment ${ }^{18-20}$. It is also obvious that misplacement of the craniotomy window over the emissary vein can result in iatrogenic morbidity, either as consequence of SSS air embolism or as a pathways by which infections are carried into the cranial cavity ${ }^{3,4}$. So, surgeons should be aware of the PF position and consider this anatomic variation.

The present study has revealed the topography of the PF in Brazilian individuals. The PF was found in $69.7 \%$ of cases, always anteriorly to the lambda, and laterally to the sagittal suture. One strength of our study is that-in order to make accurate assessments-we used contrast-enhanced MRI imaging instead of dry skulls and confirmed that vascular tethers penetrate the foramen. Little holes without emissary veins were not considered foramina in this study ${ }^{4}$. Also, we were able to compare different external landmarks to find the most stable guide to the PF.

Inion, bregma and lambda were used to determine PF location (Fig. 1 and 2) within a $20 \mathrm{~mm}$ radius from each parameter's mean value, a different PF scatter pattern was generated. $92.6 \%, 91.8 \%, 90.1 \%$ of the PFs were inside that radius when measured from the (\#1) lambda (mean $35.3 \mathrm{~mm}),(\# 5)$ inion (mean $102.8 \mathrm{~mm}$ ) and (\#4) bregma (mean $94.9 \mathrm{~mm}$ ), respectively. This could be due the different cranial trajectories implied in each measurement. An opposite relation was found when analyzing the coefficient of variance (Table 1). Bregma (8.8\%) had the smallest variation by a substantial margin, followed by inion (13.4\%) and lambda (19.6\%). Additionally, lambda is a landmark that can hardly be palpated on a 
clinical setting. This gives an advantage to both bregma and inion, as they are easily visible and palpable.

A possible reason for lambda and inion to present a bigger coefficient of variance than the more stable bregma is a large variance in the squamous part of the occipital bone's area. whose mean was $10,477 \mathrm{~mm}^{2} \pm 1,792 \mathrm{~mm}^{2}$ with a coefficient of variance $17.1 \%$, a value intermediate to inions $(13.4 \%$ ) and lambdas (19.6\%). Such variation affects lambda more than the inion, as the lambda is at the peak of the occipital bone, influenced by the whole bone height, whilst the inion is in the occipital bone's middle third. Additionally, the occasional presence of Wormian bones inside lambda can decrease this points accuracy to find the $\mathrm{PF}^{21}$.

On the other hand, bruner at al. has shown that the distance between both the paracentral lobule and bregma, and the parietal lobe and lambda are actively modified by variations in precuneal area size. Hence, the relative cranial position to the cerebral landmarks do vary as a function of the parietal area $\operatorname{size}^{22}$. These findings mean that the specific position of the cerebral parenchymal structures is sensitive to multiple factors, without necessarily a correspondence with the bone landmarks. This eventual absence of correspondence between brain and skull boundaries suggests caution when making inferences about the brain areas from the position of the cranial sutures ${ }^{23}$.

Amalgamation of this data suggests that bregma can successfully be palpated and used to find PF in a clinical environment, as it is mostly found inside the $20 \mathrm{~mm}$ radius. Additionally, when the PF is in fact inside the radius, its consistency is increased, as illustrated by the coefficient of variation. Thus, studies and procedures using bregma as reference should be prioritized.

One particularly interesting finding was that the PF and emissary veins may provide a useful landmark of the SSS, which lies immediately below. Contrast-enhanced MR imaging is useful for delineating the vascular components of the $\mathrm{PF}^{4,5,8}$. The relationship between $\mathrm{PF}$ and SSS was measured and compared to previous literature findings of the SSS-sagittal suture and SSS- midline relationships, at the posterior third of the suture ${ }^{24,25}$. The correlation between the left PF and the SSS was mostly constant, especially in the left hemisphere. $88 \%$ of the 62 left PF's were situated within $1 \mathrm{~cm}$, laterally to the left margin of the SSS. The right PF's distribution was more spread. 61.7\% were situated within $1.4 \mathrm{~cm}$ laterally from the right margin of the SSS, while $38.3 \%$ were directly above the SSS (figure 3 ).

In a 30-specimen cadaveric study, the SSS was deviated to the right of the sagittal suture in $63 \%$, laid under the suture in $20 \%$ and deviated to the left in $17 \% 24$. It appears that the relation between SSS and the sagittal suture tends to be less constant in this area of the crania when compared to PF-SSS. Another study concluded that the SSS was consistently displaced on either side of midline. Thus, the midline is not reliable for identifying the $\mathrm{SSS}^{25}$. This could mean that the PF is a more reliable landmark for predicting the SSS position and navigating this region than the sagittal suture and midline, with highlight to the left PF, especially useful to help surgeons avoid iatrogenic injuries in emergency surgeries. 
Computerized tomography technique help physician to identify both the underlying emissary vein and SSS in individual patients and so also reduce the risk of direct puncturing the vascular bundle. However, considering the possibility of a lack of reliable equipment or the presence of emergency surgeries, we suggest a simple method that would provide a convenient guideline and aide the clinician in avoid damaging the vessels and potentially fatal hemorrhage. We propose that incisions $1 \mathrm{~cm}$ laterality to PF should avoid injuring vascular structures. As even in the cases where the PF was directly above the SSS ( $11 \%$ on the left and $39 \%$ on the right), its respective margins distanced no more than $4 \mathrm{~mm}$, to the left PF and $8.5 \mathrm{~mm}$ to the right PF.

Parameter \#8 small coefficient of variance (9.6\%) suggest a vascular relationship in which the confluence of sinuses or adjunct venous structures, may influences PFs positioning, possibly through the formation of the emissary vein. This valveless vein plays an important role in regulating intracranial pressure and brain temperature. its ability to modify the direction of blood from the intracranial space to the extracranial one or vice versa. In the scalp the temperature is, most of the time, lower than in the intracranial compartment and the exchange of blood may regulate the intradural temperature. Furthermore, in patients with parasagittal meningiomas, the emissary vein can supply the tumor. The emissary vein can be used as access to embolize dural vessels ${ }^{26}$. So, Accurate localization of the PF and emissary vein on radiological examination followed by careful obstruction of the EV can lessen the intraoperative blood loss or SSS risk of injure. Thus, awareness of the PF can also lead to development of new therapeutic strategies.

The angular gyrus is a functionally asymmetric cortex, consistently activated in a variety of tasks. Damage to the left gyri has a devastating effect on word processing, while stimulation on the right, can trigger out-of-body experience ${ }^{27,28}$. Hence, careful maneuver around such area is necessary. Additionally, anatomical laterality asymmetry is also present. When measured from the obelion to angular gyrus's reference point upon sagittal suture (parameter \#3) the right angular gyrus $(34.9 \pm 10.6 \mathrm{~mm})$ is more anterior than the left $(25.6 \pm 10.1 \mathrm{~mm})$. Alternatively, the lateral distance between the Angular Gyrus's Reference point upon Sagittal Suture and the angular gyrus averaged on $75.1 \pm 12.1 \mathrm{~mm}^{18,19,29,30}$.

This study had some limitations. Namely, the number of specimens was insufficient to determine the frequency of the presence of the PF in the brazilian population. Second, our studies were performed only in Brazilian patients. Considering the anatomical variations in cranial bone amongst races, the results could differ in other specimens. Lastly, because of bony structural changes with age ${ }^{31}$, the topography of the PF as mainly obtained in elderly subjects (age mean 58.9 years) in our study could be different in young subjects, especially pediatric patients. Thus, future studies with larger samples including different age groups should investigate the frequency in regions of the world as well as racial differences.

In conclusion, considering our findings and previous reports, the high prevalence of the PF means that a surgeon should be aware of this anatomic variation when performing any surgical or invasive procedures in the parietal region. Especially in procedures that need to maneuver around the SSS, which the PF appears to be a reliable landmark. The bregma is the most stable and accurate landmark to locate the PF. 
The standardization of a method on localizing the PF can assist clinicians performing parietal craniotomies and thereby prevent iatrogenic SSS damage, and aide further studies on PF's anatomical correlations.

\section{Methods}

142 cerebral MRI examinations were studied. Only subjects without expansive intracranial processes (tumor, subacute ischemia, or hemorrhage) were reviewed. After excluding 23 anatomically disrupted patients, 119 patients were suitable for morphometric measurements, examined independently of their right and left halves. The mean age was 58.9 years (range, 18-80 years). Examinations were performed using a 1.5 T MR scanner equipped with an 8-channel head coil (Achieva02; Philips Medical Systems, Recife, Brazil). Contrast examination with intravenous infusion of gadolinium $(0.1 \mathrm{mmol} / \mathrm{kg})$ on T1weighted sequence and obtained in sagittal sections of the whole cranial vault, were performed with the following parameters: pulse repetition time $6.983 \mathrm{~ms}$, echo delay time $3.162 \mathrm{~ms}$, slice thickness $1.00 \mathrm{~mm}$, interslice gap $0.5 \mathrm{~mm}$, matrix $0256256 \mathrm{0}$, flip angle 8, and scan duration $5 \mathrm{~min} 19 \mathrm{~s}$. All imaging data in the contrast examinations were transferred to the software OsiriX 10.0.1 to be analyzed. Additionally, 20 patients of the 119 pool were randomly selected to have their squamous part of the occipital bone measured in a CT scan. In difficult instances a consensus decision was reached through discussion between the main investigator (PTB) and a senior investigator with a $>15$-year track record neuroradiologist (A.M.B). The study was approved by the Ethics in Research Involving Human Subjects Committee at the Federal University of Pernambuco, Brazil (CAAE: 79464917.6.0000.5208). All methods were performed in accordance with the relevant guidelines and regulations and informed consent was obtained when necessary.

\section{Measured parameters.}

The location of the PF was determined using the following external landmarks: obelion, inion, bregma and lambda (Fig. 1, Fig2, Fig 3 and Table 1). We measured the following parameters using OsiriX 10.0.1 software, except parameter \#12, which was measured by Slicer 4.11.0:

\# 1. Vertical distance from obelion to lambda.

\# 2. Horizontal distance from obelion to PF.

\# 3. Distance from obelion to angular gyrus Reference point upon sagittal suture.

\# 4. Distance from PF to Bregma.

\# 5. Distance from PF to Inion.

\# 6. Distance from PF to the Central sulcus.

\# 7. Distance from PF to Vertex. 
\# 8. Distance from PF to Confluence of sinuses.

\# 9. Distance from PF to Eurion.

\# 10. Distance from Angular gyrus to PF.

\# 11. Distance from Angular gyrus to Angular gyrus reference point upon sagittal suture.

\# 12. Area of the squamous part of the occipital bone

\# 13. Distances from PF to the margins of the SSS.

\section{Statistical analysis.}

Statistical analyses were performed using SPSS (version 18.0, SPSS, Chicago, IL, USA). We used the Shapiro-Wilk test for determining whether the data conformed to a parametric or nonparametric distribution. Full heads were examined in their two halves in the 83 patients and the laterality was verified using paired samples-t test. The linear relationships between the study parameters (between \#1 and \#6, \# 1 and \#5, \#1 and \#4) were calculated using Pearson's correlation test. Chi-square test was used to determine relations between PFs side distribution prevalence in males and females. Conclusions are made at $p=0.05$ level of significance.

Table 1. Measured parameters. Detailed descriptions are provided in main text and Figure 2. Units are in millimeters, except for parameter 12 (millimeters ${ }^{2}$ ) and coefficient of variances (percentages). Parameters in which paired samples t-test did not present left-right laterality significant difference were grouped $(n=122)$. Otherwise, their values are shown separately as left $(n=62)$ and right $(n=60)$. Parameter $12 n=$ 20. AGyRSS, Angular Gyrus Reference point upon Sagittal Suture; \#, number of measured parameter. * Denotes statistically significant results $\left({ }^{*} \mathrm{p}<0.01\right)$ 


\begin{tabular}{|c|c|c|c|c|c|c|}
\hline Anatomic parameter & & \# & \multicolumn{2}{|c|}{$\begin{array}{l}\text { Mean } \pm S D \\
\text { (Left } \mid \text { Right) }\end{array}$} & \multicolumn{2}{|c|}{$\begin{array}{l}\begin{array}{l}\text { Coefficient of } \\
\text { variance }\end{array} \\
\text { (Left| Right) }\end{array}$} \\
\hline \multirow[t]{5}{*}{ Distance from obelion to } & Lambda & 1 & $35.3 \pm$ & & 19.6 & \\
\hline & Parietal foramen & 2 & $8.9 \pm 3$ & & 34.7 & \\
\hline & AGyRSS** & 3 & $\begin{array}{l}25.6 \pm \\
10.6\end{array}$ & $\begin{array}{l}34.9 \pm \\
10.1\end{array}$ & 41.3 & 28.8 \\
\hline & Bregma & 4 & \multicolumn{2}{|c|}{$94,9 \pm 8.3$} & \multicolumn{2}{|l|}{8.8} \\
\hline & Inion & 5 & \multicolumn{2}{|c|}{$102.8 \pm 14.0$} & \multicolumn{2}{|l|}{13.4} \\
\hline \multirow[t]{4}{*}{ Distance from PF to } & Central Sulcus** & 6 & $\begin{array}{l}50.9 \pm \\
9.7\end{array}$ & $\begin{array}{l}43.1 \pm \\
11.4\end{array}$ & 19.1 & 26.4 \\
\hline & Vertex & 7 & \multicolumn{2}{|c|}{$73.5 \pm 14.0$} & \multicolumn{2}{|l|}{19.0} \\
\hline & $\begin{array}{l}\text { Confluence of } \\
\text { sinuses }\end{array}$ & 8 & \multicolumn{2}{|c|}{$84.9 \pm 8.2$} & \multicolumn{2}{|l|}{9.6} \\
\hline & Eurion** & 9 & $\begin{array}{l}72.0 \pm \\
7.2\end{array}$ & $\begin{array}{l}76.8 \pm \\
7.9\end{array}$ & 10.7 & 10.2 \\
\hline \multirow[t]{2}{*}{$\begin{array}{l}\text { Distance from angular } \\
\text { gyrus to }\end{array}$} & Parietal foramen $\star \star$ & 10 & $\begin{array}{l}56.9 \pm \\
11.2\end{array}$ & $\begin{array}{l}64.8 \pm \\
8.1\end{array}$ & 19.7 & 14.5 \\
\hline & AGyRSS & 11 & \multicolumn{2}{|c|}{$75.1 \pm 12.1$} & \multicolumn{2}{|l|}{16.1} \\
\hline \multicolumn{2}{|c|}{ Area of the squamous part of the occipital bone } & 12 & \multicolumn{2}{|c|}{$10,477 \pm 1,792$} & \multicolumn{2}{|l|}{17.0} \\
\hline
\end{tabular}

\section{Declarations}

\section{Data availability}

The datasets generated and analyzed during the current study are available from the corresponding author on reasonable request.

\section{Acknowledgements}

This research was supported by the institutional program of grants for scientific initiation through the Conselho Nacional de Desenvolvimento Científico e Tecnológico (CNPq) funded by the Brazilian government. Mr Austin Post for inspiring this study. Mr. João Brainer for supporting this study.

\section{Author contributions statement}

Marcelo Valença designed this study, wrote, and revised this manuscript. Pedro Brainer-Lima analyzed and interpreted the data, wrote the manuscript and illustrated all the figures. Maria Rosana Ferreira 
helped design this study and wrote it. Alessandra Brainer-Lima analyzed the data. Paulo Brainer-Lima acquired data.

\section{Additional information}

The authors declare no competing interests.

Correspondence and requests for materials should be addressed to. Reprints

\section{References}

1. Gusmão, S., Silveira, R. L. \& Arantes, A. Pontos referenciais nos acessos cranianos. Arq. Neuropsiquiatr.61, 305-308 (2003).

2. Panteado CV, S. N. H. The number and location of the parietal foramen in human skulls. Anat Anz 39-41 (1985).

3. Tsutsumi, S., Nonaka, S., Ono, H. \& Yasumoto, Y. The extracranial to intracranial anastomotic channel through the parietal foramen: delineation with magnetic resonance imaging. Surg. Radiol. Anat.38, 455-459 (2016).

4. Mortazavi, M. M. et al. Anatomy and pathology of the cranial emissary veins: A review with surgical implications. Neurosurgery70, 1312-1318 (2012).

5. Freire, A. R. et al. Emissary Foramens of the Human Skull: Anatomical Characteristics and its Relations with Clinical Neurosurgery. Int. J. Morphol.31, 287-292 (2013).

6. Tubbs, R. S., Smyth, M. D. \& Oakes, W. J. Parietal foramina are not synonymous with giant parietal foramina. Pediatr. Neurosurg.39, 216-217 (2003).

7. Currarino, G. Normal Variants in the and Region Congenital of the Anomalies Obelion. Am. J. Roentgenol.127, 487-494 (1976).

8. Yoshioka, N., Rhoton, A. L. \& Abe, H. Scalp to meningeal arterial anastomosis in the parietal foramen. Neurosurgery58, 123-126 (2006).

9. Goyena, R. \& Fallis, A. . MORFOMETRIA DOS FORAMES EMISSÁRIOS PARIETAIS: PREVALÊNCIA E VARIAÇÕES RELACIONADAS AO SEXO. J. Chem. Inf. Model.53, 1689-1699 (2019).

10. Collipal, E., Silva, H., Quintas, F., Martínez, C. \& del Sol, M. Estudio Morfométrico del Foramen Parietal. Int. J. Morphol.27, 481-484 (2009).

11. Irmak, M. K., Korkmaz, A. \& Erogul, O. Selective brain cooling seems to be a mechanism leading to human craniofacial diversity observed in different geographical regions. 974-979 (2004). doi:10.1016/j.mehy.2004.05.003

12. Cabanac, M. \& Brinnel, H. Applied Physiology Blood flow in the emissary veins of the human head during hyperthermia. 172-176 (1985).

13. Falk, D. Evolution of Cranial Blood Drainage in Hominids: Enlarged Occipital / Marginal Sinuses and Emissary Foramina. 324, 311-324 (1986). 
14. Reviews, B. Homo erectus. 90-92 (1990).

15. Cotton, F. et al. Cranial sutures and craniometric points detected on MRI. Surg. Radiol. Anat.27, 6470 (2005).

16. Salamon, N., Sicotte, N., Mongkolwat, P., Shattuck, D. \& Salamon, G. The human cerebral cortex on MRI: Value of the coronal plane. Surg. Radiol. Anat.27, 431-443 (2005).

17. Juerchott, A. et al. 3D cephalometric analysis using Magnetic Resonance Imaging: validation of accuracy and reproducibility. Sci. Rep.8, 1-11 (2018).

18. Campero, A. et al. Brain sulci and gyri: A practical anatomical review. J. Clin. Neurosci.21, 22192225 (2014).

19. Wild, H. M., Heckemann, R. A., Studholme, C. \& Hammers, A. Gyri of the human parietal lobe: Volumes, spatial extents, automatic labelling, and probabilistic atlases. PLoS One12, 1-28 (2017).

20. Ahdab, R. et al. Reappraisal of the anatomical landmarks of motor and premotor cortical regions for image-guided brain navigation in TMS practice. Hum. Brain Mapp.35, 2435-2447 (2014).

21. Kumar T.M, V., Kumar, V. \& Yadav, N. the Occurrence of Wormian Bones Within the Cranial Sutures and Their Clinical Significance. Int. J. Anat. Res.4, 3082-3086 (2016).

22. Bruner, E., Amano, H., de la Cuétara, J. M. \& Ogihara, N. The brain and the braincase: A spatial analysis on the midsagittal profile in adult humans. J. Anat.227, 268-276 (2015).

23. Jiang, X., Iseki, S., Maxson, R. E., Sucov, H. M. \& Morriss-Kay, G. M. Tissue origins and interactions in the mammalian skull vault. Dev. Biol.241, 106-116 (2002).

24. Tubbs, R. S., Salter, G., Elton, S., Grabb, P. A. \& Oakes, W. J. Sagittal suture as an external landmark for the superior sagittal sinus. J. Neurosurg.94, 985-987 (2001).

25. Reis, C. V. C. et al. Midline as a landmark for the position of the superior sagittal sinus on the cranial vault: An anatomical and imaging study. Surg. Neurol. Int.6, (2015).

26. Nawashiro, H., Nawashiro, T. \& Nawashiro, A. Subcutaneous Extension of Parasagittal Atypical Meningioma Through Parietal Foramen. World Neurosurg.125, 104-105 (2019).

27. Seghier, M. L. The angular gyrus: Multiple functions and multiple subdivisions. Neuroscientist19, 4361 (2013).

28. Kurimoto, M. et al. Safe removal of glioblastoma near the angular gyrus by awake surgery preserving calculation ability - Case report. Neurol. Med. Chir. (Tokyo).46, 46-50 (2006).

29. Rusconi, E. Gerstmann syndrome: historic and current perspectives. Handbook of Clinical Neurology151, (Elsevier B.V., 2018).

30. Lerch, J. P. et al. Studying neuroanatomy using MRI. Nat. Neurosci.20, 314-326 (2017).

31. Urban, J. E. et al. Evaluation of morphological changes in the adult skull with age and sex. J. Anat.229, 838-846 (2016).

\section{Figures}



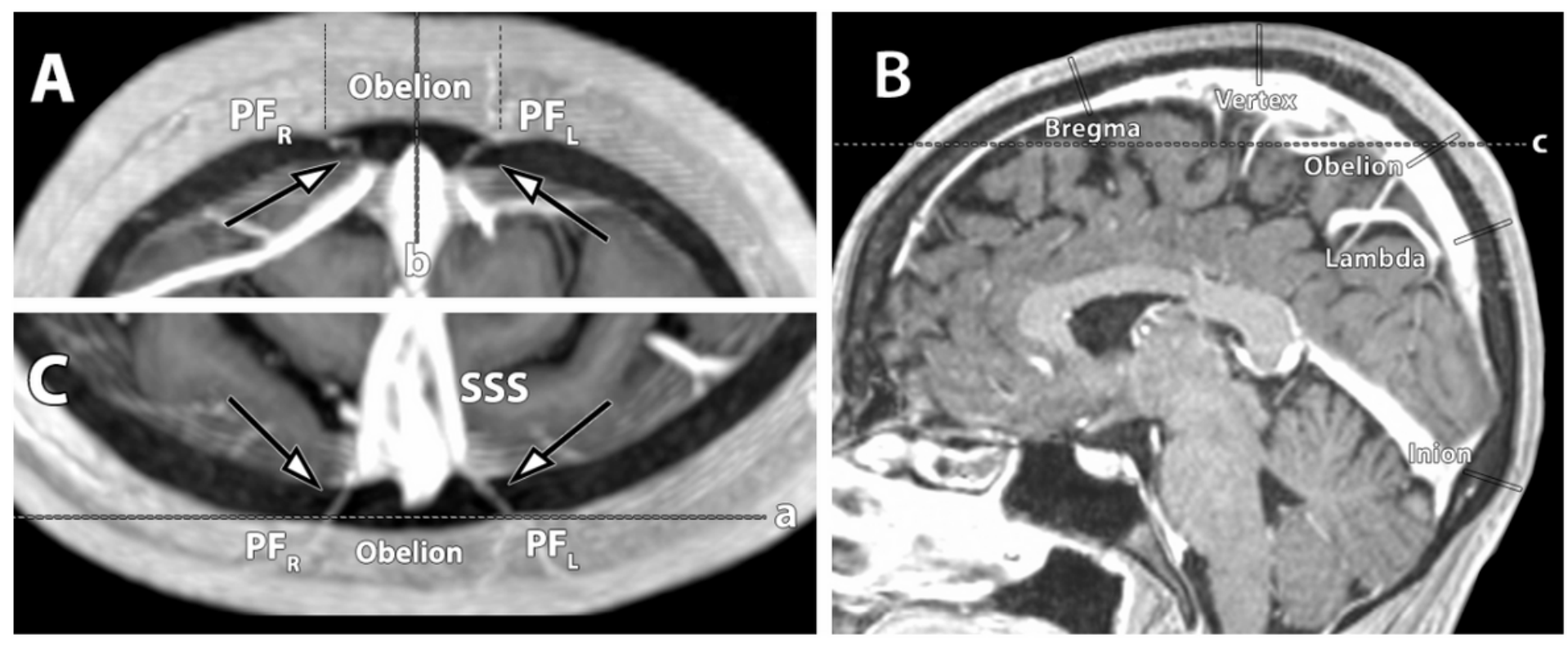

Figure 1

MRI studies demonstrating cranial and vascular landmarks. A. $5 \mathrm{~mm}$ thickness Maximum intensity projection (MIP) of coronal slice, exhibiting simetrical right (PFR) and left (PFL) Parietal foramina and emissary veins (arrows), mirroed around the obelion. B. Midline sagittal slice demonstrating spacial disposition between main midline skull landmarks. C. $5 \mathrm{~mm}$ thickness MIP of Axial slice, showing emissary veins converging to the superior sagittal sinus (SSS). Dotted line represents slice level of represented letter. ' $a$,', $b$ ' and ' $c$ ' represents the respective heights of the slice where images 1A, 1B and 1C were accquired. 


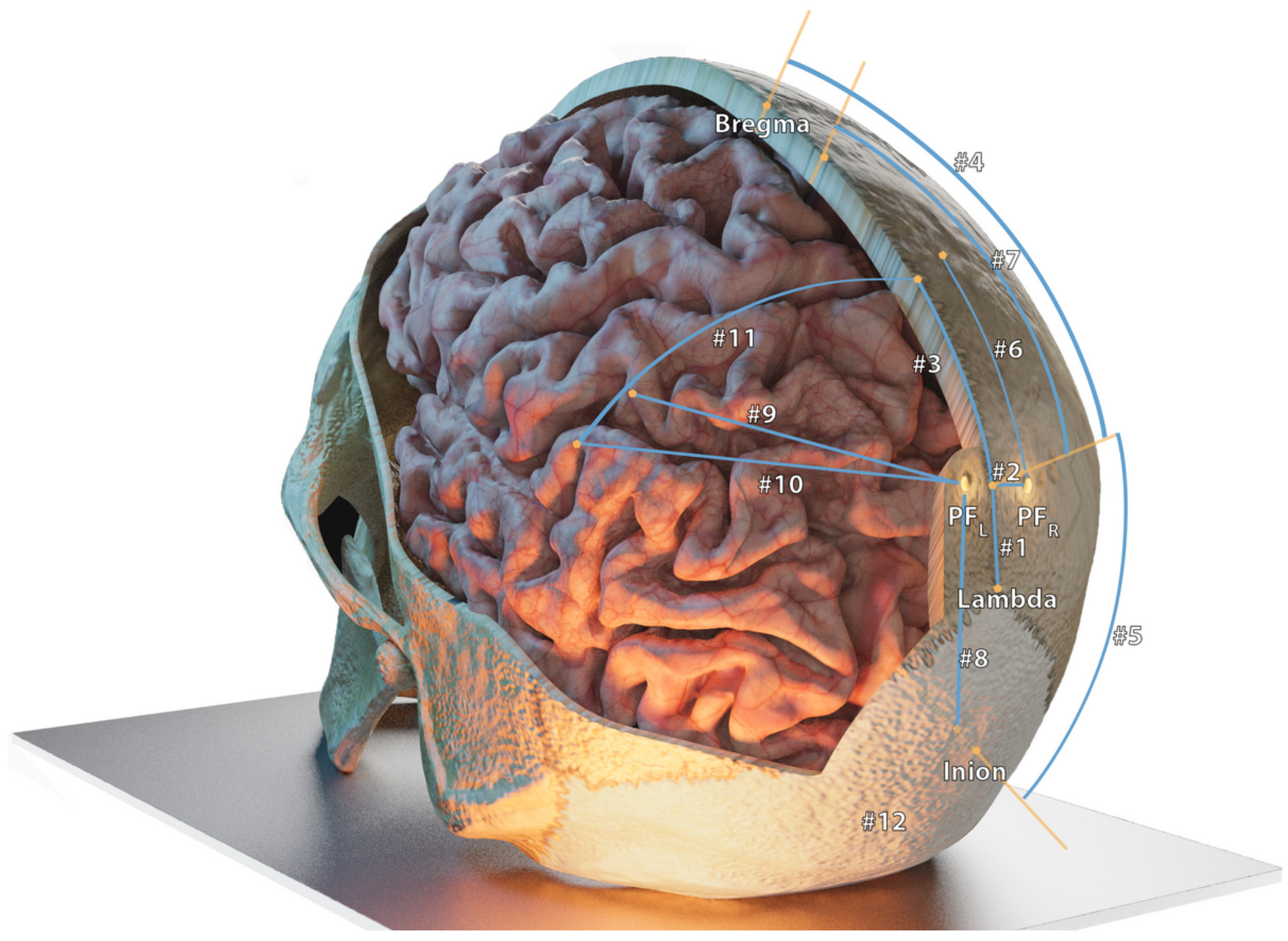

Figure 2

Illustration of the main parameters (\#1 to \#12). PF, Parietal foramen. Right Parietal Foramen (PFR) and Left (PFL). 


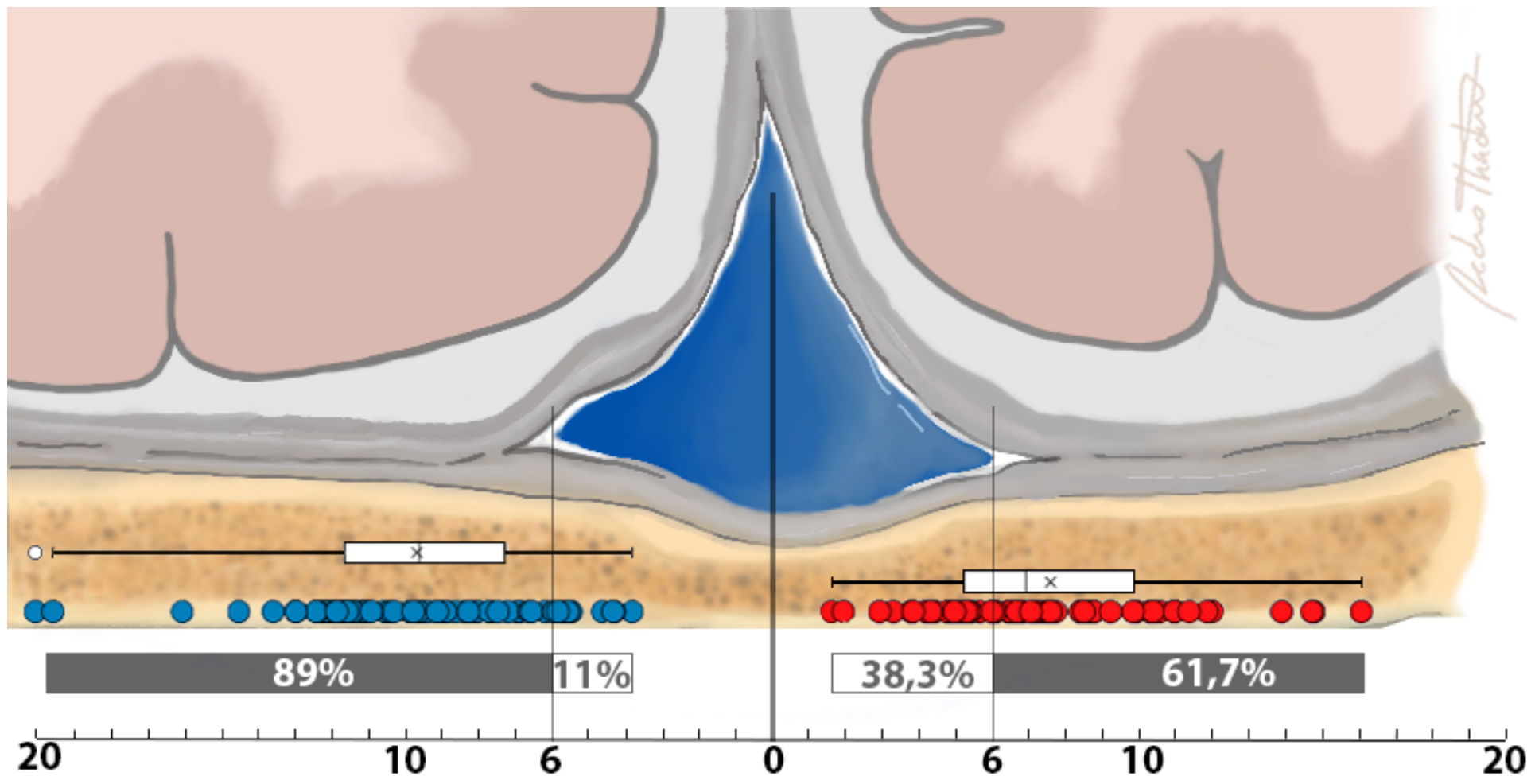

Figure 3

Illustration of the Parietal foramina external opening dispersion, and its relation to the superior sagittal sinus. $89 \%$ of the left PFs was within a $1.4 \mathrm{~cm}$ distance, laterally to the left margin of the SSS, while $11 \%$ were directly above the SSS. $61.7 \%$ of the right PFs were laterally to the right margin of the SSS, while $38.3 \%$ were directly above it. Units: Millimeters. ' $X$ ' = mean, 'I' Median.

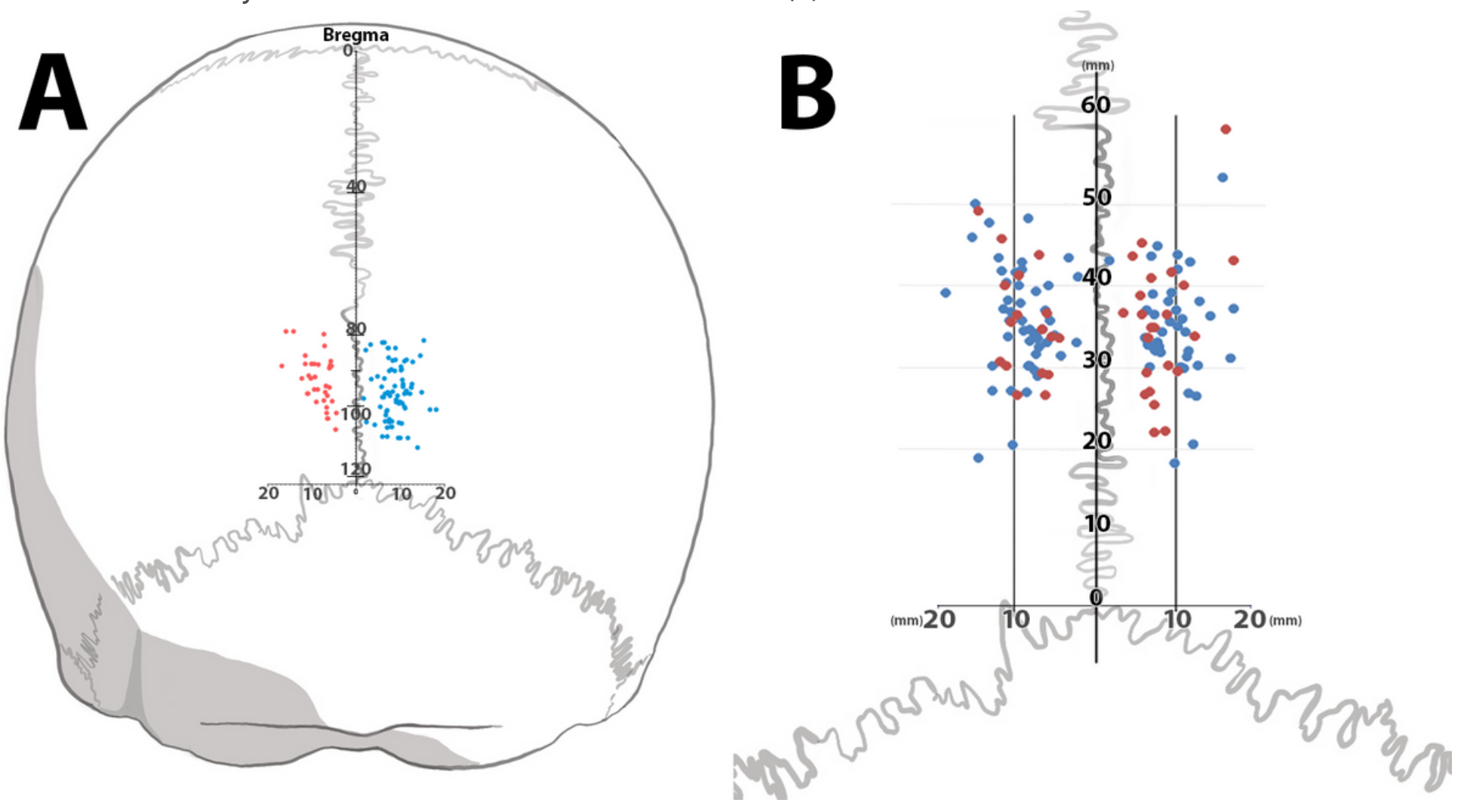




\section{Figure 4}

Schematic drawing of the distribution of the PF. No distribution pattern differences were found between man and woman. Figure 4A compares the bregma-measured PF distribution difference between men (blue) and women(red). Measures are presented in this image regardless of laterality differences. Figure 4B compares right and left PFs distribution, in which female (red) and male (blue). $92.6 \%$ of PFs were located within a $20 \mathrm{~mm}$ radius from the mean value. Unit: millimeters. 